\title{
SUCCESSIVE APPROXIMATIONS AND INTERVAL HALVING FOR INTEGRAL BOUNDARY VALUE PROBLEMS
}

\author{
M. RONTÓ AND Y. VARHA
}

Received 25 May, 2015

\begin{abstract}
We show how a suitable interval halving and parametrization technique can help to essentially improve the sufficient convergence condition for the successive approximations dealing with solutions of nonlinear non-autonomous systems of ordinary differential equations under integral boundary conditions.
\end{abstract}

2010 Mathematics Subject Classification: 34B15

Keywords: integral boundary value problems, parametrization technique, successive approximations, interval halving

\section{INTRODUCTION}

Recently, boundary value problems (BVPs) with integral conditions for non-linear differential equations have attracted much attention, see, e.g. [1],[2] and the references therein. However mainly scalar non-linear differential equations of special kinds have been studied. According to our best knowledge, there are only a few works dealing with a constructive investigation of systems of non-linear differential equations of a general form with linear or non-linear integral boundary restrictions. So, in [10] the following mixed integral BVP was studied

$$
\begin{gathered}
\frac{d x(t)}{d t}=f(t, x(t)), t \in[0, T], \\
A x(0)+\int_{0}^{T} P(s) x(s) d s+C x(T)=d .
\end{gathered}
$$

The paper [6] deals with the problem of general form

$$
\begin{gathered}
\frac{d u(t)}{d t}=f(t, u(t)), t \in[a, b], \\
\Phi(u)=d,
\end{gathered}
$$

where $\Phi: C\left([a, b], \mathbb{R}^{n}\right)$ is a vector functional (possibly non-linear), $f:[a, b] \times \mathbb{R}^{n} \rightarrow$ $\mathbb{R}^{n}$ is a function satisfying the Caratheodory condition in a certain bounded set and 
$d$ is a given vector. In [9] the authors investigated the problem for which the integral boundary restrictions also depend upon the derivative of the solution

$$
\begin{gathered}
\frac{d u(t)}{d t}=f(t, u(t)), t \in[a, b], \\
\int_{a}^{b}[g(s, x(s))+h(s, f(s, x(s)))] d s=d
\end{gathered}
$$

and in [5] it was given a constructive existence analysis of solutions of the problem above.

Note, that the investigation of the solutions of problems (1.1) and (1.2) was connected with the properties of the following special sequence of functions well posed on the interval $t \in[a, b]$

$$
\begin{gathered}
x_{0}(t, z, \eta):=z+\frac{t-a}{b-a}[\eta-z]=\left[1-\frac{t-a}{b-a}\right] z+\frac{t-a}{b-a} \eta, t \in[a, b], \\
x_{m}(t, z, \eta):=z+\int_{a}^{t} f\left(s, x_{m-1}(s, z, \eta)\right) d s- \\
-\frac{t-a}{b-a} \int_{a}^{b} f\left(s, x_{m-1}(s, z, \eta)\right) d s+\frac{t-a}{b-a}[\eta-z], t \in[a, b],
\end{gathered}
$$

where $z$ and $\eta$ are some vector-parameters.

The aim of this paper is to show how a natural interval halving and parametrization technique can help to essentially improve the sufficient convergence conditions mentioned in papers [6], [9].

More precisely we consider the problem

$$
\begin{gathered}
\frac{d u(t)}{d t}=f(t, u(t)), t \in[a, b], \\
\int_{a}^{b} g(s, x(s)) d s=d,
\end{gathered}
$$

where $f:[a, b] \times D \rightarrow \mathbb{R}^{n}, g:[a, b] \times D \rightarrow \mathbb{R}^{n}$ are a continuous functions in a certain bounded set $D$ and $d \in \mathbb{R}^{n}$ is a given vector.

We use an appropriate numerical-analytic approach and a natural interval halving technique which was suggested in [6], [4], [7], [8],[11], [3]. At first, we reduce 
the given problem (1.4), (1.5) to two "model-type" two-point BVPs with separated parameterized conditions

$$
\begin{gathered}
\frac{d x}{d t}=f(t, x), t \in\left[a, a+\frac{b-a}{2}\right], \\
x(a)=z, x\left(a+\frac{b-a}{2}\right)=\lambda
\end{gathered}
$$

and

$$
\begin{gathered}
\frac{d y}{d t}=f(t, y), t \in\left[a+\frac{b-a}{2}, b\right], \\
y\left(a+\frac{b-a}{2}\right)=\lambda, y(b)=\eta,
\end{gathered}
$$

where $z, \lambda, \eta \in \mathbb{R}^{n}$ are parameters. Note, that the length of the interval in problems (1.6), (1.7) and (1.8), (1.9) is $\frac{b-a}{2}$ opposite to $b-a$ in the case of original BVP (1.4), (1.5).

To study the solutions of BVPs (1.6), (1.7) and (1.8), (1.9) we use the special modified form of parameterized successive approximations $x_{m}(t, z, \lambda)$ and $y_{m}(t, \lambda, \eta)$ of type (1.3) constructed in analytic form and well defined on the intervals $t \in\left[a, a+\frac{b-a}{2}\right]$ and $t \in\left[a+\frac{b-a}{2}, b\right]$, respectively.

We give sufficient conditions for the uniform convergence of these successive approximations to some limit functions $x_{\infty}(t, z, \lambda)$ and $y_{\infty}(t, \lambda, \eta)$, respectively. The main limitation in order to guarantee the convergence of the introduced sequences $\left\{x_{m}(t, z, \lambda)\right\}_{m=0}^{\infty}$ and $\left\{y_{m}(t, z, \lambda)\right\}_{m=0}^{\infty}$ is that one has to assume a certain smallness of the eigenvalue $r(Q)$ of the matrix $Q:=\frac{3(b-a)}{20} K$, where $K$ stands in the Lipschitz condition $\left|f\left(t, u_{1}\right)-f\left(t, u_{2}\right)\right| \leq K\left|u_{1}-u_{2}\right|$, provided that

$$
r(Q)<1 \text {. }
$$

Note that in [6], [9], [5], [10] where the numerical-analytic approach was applied, instead of the previous inequality the convergence condition

$$
2 r(Q)<1 .
$$

has appeared. Thus, the convergence condition is weakened by its half. We show that the limit functions $x_{\infty}(t, z, \lambda)$ and $y_{\infty}(t, \lambda, \eta)$ are solutions of some additively modified equations of form (1.6) and (1.8). The functional perturbation term, by which the modified equation differs from the original one, essentially depends on the parameters $z, \lambda, \eta$ and generates finitely many determining equations together with (1.5) from which the numerical values of these parameters should be found. 


\section{NOTATION AND DEFINITIONS}

We fix an $n \in \mathbb{N}$ and a bounded set $D \subset \mathbb{R}^{n}$. The following symbols are used in the sequel:

1. For vectors $x=\operatorname{col}\left(x_{1}, \ldots, x_{n}\right) \in \mathbb{R}^{n}$ the obvious notation $|x|=\operatorname{col}\left(\left|x_{1}\right|, \ldots,\left|x_{n}\right|\right)$ is used and the inequalities between vectors are understood component-wise.

The same convention is adopted implicitly for operations ' $\max ^{\prime},{ }^{\prime} \min ^{\prime}$, ' sup $^{\prime}$, ' inf $^{\prime}$, so that e.g. $\max \{h(z): z \in Q\}$ for any $h=\left(h_{i}\right)_{i=1}^{n}: Q \rightarrow \mathbb{R}^{n}$, where $Q \subset \mathbb{R}^{m}$, $m \leq n$, is defined as the column vector with components $\max \left\{h_{i}(z): z \in Q\right\}, i=$ $1,2, \ldots, n$.

2. $1_{n}$ is the unit matrix of dimension $n$.

3. $0_{n}$ is the zero matrix of dimension $n$.

4. $r(K)$ is the maximal eigenvalue (in modulus) of a matrix $K$.

Definition 1. For any non-negative vector $\rho \in \mathbb{R}^{n}$ under the component-wise $\rho$-neighborhood of a point $z \in \mathbb{R}^{n}$ we understand

$$
B(z, \rho):=\left\{\xi \in \mathbb{R}^{n}:|\xi-z| \leq \rho\right\} .
$$

Similarly, for the given bounded connected set $\Omega \subset \mathbb{R}^{n}$, we define its componentwise $\rho-$ neighborhood by putting

$$
B(\Omega, \rho):=\underset{\xi \in \Omega}{\cup} B(\xi, \rho) .
$$

Definition 2. For given two bounded connected sets $D_{a} \subset \mathbb{R}^{n}$ and $D_{b} \subset \mathbb{R}^{n}$, introduce the set

$$
D_{a, b}:=(1-\theta) z+\theta \eta, z \in D_{a}, \eta \in D_{b}, \theta \in[0,1]
$$

and its component-wise $\rho$-neighborhood

$$
D:=B\left(D_{a, b}, \rho\right) .
$$

For a set $D \subset \mathbb{R}^{n}$, closed interval $[a, b] \subset \mathbb{R}$, continuous function $f:[a, b] \times D \rightarrow$ $\mathbb{R}^{n}, n \times n$ matrix $K$ with non-negative entries, we write

$$
f \in \operatorname{Lip}(K, D)
$$

if the inequality

$$
|f(t, u)-f(t, v)| \leq K|u-v|
$$

holds for all $\{u, v\} \subset D$ and $t \in[a, b]$.

Finally, on the base of function $f:[a, b] \times D \rightarrow \mathbb{R}^{n}$ we introduce the vector

$$
\delta_{[a, b], D}(f):=\frac{1}{2}\left[\max _{(t, x) \in[a, b] \times D} f(t, x)-\min _{(t, x) \in[a, b] \times D} f(t, x)\right] .
$$




\section{PROBlem SETTING AND REDUCTION TO MODEL-TYPE, SUBSIDIARY STATEMENTS}

Let us consider the nonlinear integral BVP (1.4), (1.5).

Let us fix certain closed bounded sets $D_{a}, D_{\frac{a+b}{2}}, D_{b} \subset \mathbb{R}^{n}$ and focus on the continuously differentiable solutions $u$ of problem (1.4), (1.5) with values

$$
u(a) \in D_{a}, u\left(\frac{a+b}{2}\right) \in D_{\frac{a+b}{2}} \text { and } u(b) \in D_{b} .
$$

Without loss of generality, we shall choose $D_{a}, D_{\frac{a+b}{2}}, D_{b}$ to be convex.

Based on the sets $D_{a}$ and $D_{\frac{a+b}{2}}$ we introduce the set

$$
D_{a, \frac{a+b}{2}}:=(1-\theta) z+\theta \lambda, z \in D_{a}, \lambda \in D_{\frac{a+b}{2}}, \theta \in[0,1],
$$

according to (2.3) and its component-wise $\rho^{x}$-neighborhood

$$
D^{x}:=B\left(D_{a, \frac{a+b}{2}}, \rho^{x}\right)
$$

as in (2.4). Similarly, based on the sets $D_{\frac{a+b}{2}}$ and $D_{b}$ we introduce the set

$$
D_{\frac{a+b}{2}, b}:=(1-\theta) \lambda+\theta \eta, \lambda \in D_{\frac{a+b}{2}}, \eta \in D_{b}, \theta \in[0,1],
$$

according to (2.3) and its component-wise $\rho^{y}$-neighborhood

$$
D^{y}:=B\left(D_{\frac{a+b}{2}, b}, \rho^{y}\right)
$$

as in (2.4).

It is important to emphasize that $D^{x}, D^{y}$ and $D$ are bounded sets and, thus, the Lipschitz conditions of type (2.5), (2.6) are not assumed globally. The problem is to find a continuously differentiable solution $u:[a, b] \rightarrow D$ of the problem (1.4), (1.5) for which inclusions (3.1) hold.

At first we simplify the boundary integral conditions (1.5) and reduce it to some two-point separated conditions. To replace the boundary conditions (1.5) by certain linear two-point linear separated ones, similarly to [6], [10], [11],[9] we apply a certain "freezing" technique. Namely, we introduce the vectors of parameters

$$
z=\operatorname{col}\left(z_{1}, z_{2}, \ldots, z_{n}\right), \lambda=\operatorname{col}\left(\lambda_{1}, \lambda_{2}, \ldots, \lambda_{n}\right), \eta=\operatorname{col}\left(\eta_{1}, \eta_{2}, \ldots, \eta_{n}\right)
$$

by formally putting

$$
z:=u(a), \lambda:=u\left(\frac{a+b}{2}\right), \eta:=u(b) .
$$

Now, instead of boundary value problem (1.4), (1.5) using a natural interval halving technique, we will consider on the intervals $t \in\left[a, \frac{a+b}{2}\right]$ and $t \in\left[a+\frac{b-a}{2}, b\right]$, respectively, the following two "model-type" two-point BVPs with separated parameterized conditions (1.6), (1.7) and (1.8), (1.9). 
The parametrization technique that we are going to use suggests that instead of the original boundary value problem (1.4) (1.5), we study the family of parameterized boundary value problems (1.6), (1.7) and (1.8), (1.9) where the boundary restrictions are linear and separated. We then go back to the original problem by choosing the values of the introduced parameters appropriately.

Remark 1. The set of solutions of the boundary value problem (1.4), (1.5) coincides with the set of the solutions of the parameterized problems (1.6), (1.7) and (1.8), (1.9) with separated restrictions, satisfying additional conditions (3.7).

We recall some subsidiary statements which are needed below in the following form.

Lemma 1 ([3], Lemma 3.13). Let $f:[\tau, \tau+I] \rightarrow \mathbb{R}^{n}$ be a continuous function. Then, for an arbitrary $t \in[\tau, \tau+I]$, the inequality

$$
\left|\int_{\tau}^{t}\left[f(\tau)-\frac{1}{I} \int_{\tau}^{\tau+I} f(s) d s\right] d \tau\right| \leq \alpha_{1}(t, \tau, I) \delta_{[\tau, \tau+I]}(f)
$$

holds, where

$$
\alpha_{1}(t, \tau, I)=2(t-\tau)\left(1-\frac{t-\tau}{I}\right),\left|\alpha_{1}(t, \tau, I)\right| \leq \frac{I}{2}, t \in[\tau, \tau+I],
$$

and

$$
\delta_{[\tau, \tau+I]}(f)=\frac{\max _{t \in[\tau, \tau+I]} f(t)-\min _{t \in[\tau, \tau+I]} f(t)}{2} .
$$

Lemma 2 ([3], Lemma 3.16). Let the sequence of continuous functions $\left\{\alpha_{m}(t, \tau, I)\right\}_{m=0}^{\infty}$, for $t \in[\tau, \tau+I]$ be defined by the recurrence relation

$$
\begin{aligned}
\alpha_{m+1}(t, \tau, I)=\left(1-\frac{t-\tau}{I}\right) \int_{\tau}^{t} & \alpha_{m}(s, \tau, I) d s+ \\
& +\frac{t-\tau}{I} \int_{t}^{\tau+I} \alpha_{m}(s, \tau, I) d s, m=0,1,2, \ldots .
\end{aligned}
$$

where

$$
\alpha_{0}(t, \tau, I)=1 .
$$

Then the following estimates hold for $t \in[\tau, \tau+I]$ :

$$
\begin{gathered}
\alpha_{m+1}(t, \tau, I) \leq \frac{10}{9}\left(\frac{3 I}{10}\right)^{m} \alpha_{1}(t, \tau, I), m \geqslant 0, \\
\alpha_{m+1}(t, \tau, I) \leq \frac{3 I}{10} \alpha_{m}(t, \tau, I), m \geqslant 2,
\end{gathered}
$$


where $\alpha_{1}(t, \tau, I)$ is given in (3.9).

\section{INTERVAL HALVING AND SUCCESSIVE APPROXIMATIONS}

So, our approach to the integral BVP (1.4), (1.5) requires that we first study the auxiliary problems (1.6), (1.7) and (1.8), (1.9) separately, for which purpose appropriate iteration processes were introduced .

Let us put that the domain of the space of variables of the problem (1.6), (1.7) is $D^{x}$.

We suppose that $f \in \operatorname{Lip}\left(K_{x}, D^{x}\right)$ with $\rho^{x}$ satisfies the inequality

$$
\rho^{x} \geq \frac{b-a}{4} \delta_{\left[a, \frac{a+b}{2}\right], D^{x}}(f)
$$

and

$$
r\left(Q_{x}\right)<1, Q_{x}=\frac{3(b-a)}{20} K_{x} .
$$

Let us define for the parameterized problem (1.6), (1.7) the recurrence parameterized sequence of functions $x_{m}:\left[a, \frac{a+b}{2}\right] \times \mathbb{R}^{n} \times \mathbb{R}^{n} \rightarrow \mathbb{R}^{n}, m=0,1,2, \ldots$, by putting

$$
\begin{gathered}
x_{0}(t, z, \lambda):=z+\frac{2(t-a)}{b-a}[\lambda-z]= \\
=\left[1-\frac{2(t-a)}{b-a}\right] z+\frac{2(t-a)}{b-a} \lambda, t \in\left[a, \frac{a+b}{2}\right], \\
x_{m}(t, z, \lambda):=z+\int_{a}^{t} f\left(s, x_{m-1}(s, z, \lambda)\right) d s- \\
-\frac{2(t-a)}{b-a} \int_{a}^{\frac{a+b}{2}} f\left(s, x_{m-1}(s, z, \lambda)\right) d s+\frac{2(t-a)}{b-a}[\lambda-z], t \in\left[a, \frac{a+b}{2}\right],
\end{gathered}
$$

for all $m=1,2, \ldots, z \in \mathbb{R}^{n}$ and $\lambda \in \mathbb{R}^{n}$.

In a similar manner, for the parameterized problem (1.8), (1.9) on the interval $\left[\frac{a+b}{2}, b\right]$, we put that the domain of the space variables for the problem is $D^{y}$ and suppose that $f \in \operatorname{Lip}\left(K_{y}, D^{y}\right)$ with $\rho^{y}$ satisfying the inequality

$$
\rho^{y} \geq \frac{b-a}{4} \delta_{\left[\frac{a+b}{2}, b\right], D^{y}}(f)
$$

and

$$
r\left(\frac{3(b-a)}{20} K_{y}\right)<1
$$


Let us define for the problem (1.8), (1.9) the recurrence parameterized sequence of functions $y_{m}:\left[\frac{a+b}{2}, b\right] \times \mathbb{R}^{n} \times \mathbb{R}^{n} \rightarrow \mathbb{R}^{n}, m=0,1,2, \ldots$, by putting

$$
\begin{gathered}
y_{0}(t, \lambda, \eta):=\lambda+\frac{2 t-a-b}{2 b-a-b}[\eta-\lambda]= \\
=\left[1-\frac{2 t-a-b}{2 b-a-b}\right] \lambda+\frac{2 t-a-b}{2 b-a-b} \eta, t \in\left[\frac{a+b}{2}, b\right] \\
y_{m}(t, \lambda, \eta):=\lambda+\int_{\frac{a+b}{2}}^{t} f\left(s, y_{m-1}(s, \lambda, \eta)\right) d s- \\
-\frac{2 t-a-b}{2 b-a-b} \int_{\frac{a+b}{2}}^{b} f\left(s, y_{m-1}(s, z, \lambda)\right) d s+\frac{2 t-a-b}{2 b-a-b}[\eta-\lambda], t \in\left[\frac{a+b}{2}, b\right]
\end{gathered}
$$

for all $m=1,2, \ldots, \lambda \in \mathbb{R}^{n}$ and $\eta \in \mathbb{R}^{n}$.

We note that all members of the sequences (4.4), (4.8) satisfy the two-point boundary conditions (1.7) and (1.9) for any $z, \lambda$ and $\eta$ from $\mathbb{R}^{n}$.

\section{CONVERGENCE OF SUCCESSIVE APPROXIMATIONS}

We would like to use the sequences $x_{m}:\left[a, \frac{a+b}{2}\right] \times \mathbb{R}^{n} \times \mathbb{R}^{n} \rightarrow \mathbb{R}^{n}, m=0,1,2, \ldots$, and $y_{m}:\left[\frac{a+b}{2}, b\right] \times \mathbb{R}^{n} \times \mathbb{R}^{n} \rightarrow \mathbb{R}^{n}, m=0,1,2, \ldots$ from (4.4) and (4.8) for the investigation of the solutions of the given boundary value problem (1.4), (1.5).

The following statement shows that the sequence (4.4) is uniformly convergent and its limit is a solution of a certain additively perturbed problem for all $(z, \lambda) \in$ $D_{a} \times D_{\frac{a+b}{2}}$.

Theorem 1. Let there exist a non negative vector $\rho^{x}$ such that $f \in \operatorname{Lip}\left(K_{x}, D^{x}\right)$ on the interval $t \in\left[a, \frac{a+b}{2}\right]$ with $\rho^{x}$ satisfying the inequality (4.7) and for the matrix

$$
Q_{x}:=\frac{3(b-a)}{20} K_{x},
$$

the inequality (5.2) is satisfied

$$
r\left(Q_{x}\right)<1 .
$$

Then, for arbitrary fixed pair of vectors $(z, \lambda) \in D_{a} \times D_{\frac{a+b}{2}}$ :

1. All members of sequence (4.4) are continuously differentiable functions on the interval $t \in\left[a, \frac{a+b}{2}\right]$ satisfying the two-point separated parameterized boundary conditions (1.7). 
2. The sequence of functions (4.4) in $t \in\left[a, \frac{a+b}{2}\right]$ converges uniformly as $m \rightarrow \infty$ to the limit function

$$
x_{\infty}(t, z, \lambda)=\lim _{m \rightarrow \infty} x_{m}(t, z, \lambda) .
$$

3. The limit function satisfies the conditions

$$
x_{\infty}(a, z, \lambda)=z, x_{\infty}\left(\frac{a+b}{2}, z, \lambda\right)=\lambda .
$$

4. The function $x_{\infty}(t, z, \lambda)$ is a unique continuously differentiable solution of the integral equation

$$
\begin{gathered}
x(t)=z+\int_{a}^{t} f(s, x(s)) d s- \\
-\frac{2(t-a)}{b-a} \int_{a}^{\frac{a+b}{2}} f(s, x(s)) d s+\frac{2(t-a)}{b-a}[\lambda-z], t \in\left[a, \frac{a+b}{2}\right]
\end{gathered}
$$

in the domain $D^{x}$. In other words, $x_{\infty}(t, z, \lambda)$ is a solution of the following Cauchy problem for the modified system of integro-differential equations:

$$
\begin{gathered}
\frac{d x}{d t}=f(t, x)+\frac{2}{b-a} \Delta(z, \lambda), t \in\left[a, \frac{a+b}{2}\right], \\
x(a)=z,
\end{gathered}
$$

where $\Delta(z, \lambda): D_{a} \times D_{\frac{a+b}{2}} \rightarrow \mathbb{R}^{n}$ is a mapping given by the formula

$$
\Delta(z, \lambda)=\lambda-z-\int_{a}^{\frac{a+b}{2}} f\left(s, x_{\infty}(s, z, \lambda)\right) d s .
$$

5. The following estimate holds:

$$
\begin{gathered}
\left|x_{\infty}(t, z, \lambda)-x_{m}(t, z, \lambda)\right| \leqslant \\
\leqslant \frac{10}{9} \alpha_{1}\left(t, a, \frac{b-a}{2}\right) Q_{x}^{m}\left(1_{n}-Q_{x}\right)^{-1} \delta_{\left[a, \frac{a+b}{2}\right] \times D^{x}}(f), t \in\left[a, \frac{a+b}{2}\right], m \geq 0,
\end{gathered}
$$

where $\delta_{\left[a, \frac{a+b}{2}\right] \times D^{x}}(f)$ is given in (2.7) and $\alpha_{1}\left(t, a, \frac{b-a}{2}\right)$ is defined by (3.9).

Proof. The validity of assertion 1 is verified by direct computation. To obtain the other required properties, similarly to [6],[9] we will prove that under the condition assumed for fixed $z \in D_{a}, \lambda \in D_{\frac{a+b}{2}}$ and $t \in\left[a, \frac{a+b}{2}\right]$ the functions of the sequence (4.4) are contained in the domain $D^{x}$ and (4.4) is a Cauchy sequence in the Banach space $C\left(\left[a, \frac{a+b}{2}\right], \mathbb{R}^{n}\right)$ equipped with the standard uniform norm. Indeed, using the 
estimate (3.8) of Lemma 1 for $\tau=a, I=\frac{b-a}{2}$, relation (4.4) for $m=0, t \in\left[a, \frac{a+b}{2}\right]$ implies that

$$
\begin{gathered}
\left|x_{1}(t, z, \lambda)-x_{0}(t, z, \lambda)\right| \leq \\
\leq \frac{1}{2} \alpha_{1}\left(t, a, \frac{a+b}{2}\right)\left[\max _{t \in[a, b]} f\left(t, x_{0}(t, z, \lambda)\right)-\min _{t \in[a, b]} f\left(t, x_{0}(t, z, \lambda)\right)\right] \\
\leq \alpha_{1}\left(t, a, \frac{a+b}{2}\right) \delta\left[a, \frac{a+b}{2}\right], D^{x}(f) \leq \frac{b-a}{4} \delta_{\left[a, \frac{a+b}{2}\right], D^{x}}(f),
\end{gathered}
$$

which means taking into account (4.1), that $x_{1}(t, z, \lambda) \in D^{x}$, whenever $(t, z, \lambda) \in$ $\left[a, \frac{a+b}{2}\right] \times D_{a} \times D_{\frac{a+b}{2}}$.

Using this and arguing by induction according to Lemma 1 we can easily establish that

$$
\begin{aligned}
\left|x_{m}(t, z, \lambda)-x_{0}(t, z, \lambda)\right| \leq \alpha_{1}(t, & \left.a, \frac{a+b}{2}\right) \delta_{\left[a, \frac{a+b}{2}\right], D^{x}}(f) \leq \\
& \leq \frac{b-a}{4} \delta_{\left[a, \frac{a+b}{2}\right], D^{x}}(f), m=2,3, \ldots,
\end{aligned}
$$

which means that all functions in (4.4) are also contained in the domain $D^{x}$, for all $m=1,2,3,$. and $(t, z, \lambda) \in\left[a, \frac{a+b}{2}\right] \times D_{a} \times D_{\frac{a+b}{2}}$.

Now, consider the difference of functions

$$
\begin{gathered}
x_{m+1}(t, z, \lambda)-x_{m}(t, z, \lambda)= \\
=\int_{a}^{t}\left[f\left(s, x_{m}(s, z, \lambda)\right)-f\left(s, x_{m-1}(s, z, \lambda)\right)\right] d s- \\
-\frac{2(t-a)}{b-a} \int_{a}^{\frac{a+b}{2}}\left[f\left(s, x_{m}(s, z, \lambda)\right)-f\left(s, x_{m-1}(s, z, \lambda)\right)\right] d s, m=1,2, \ldots
\end{gathered}
$$

and introduce the notation

$$
r_{m}(t, z, \lambda):=\left|x_{m}(t, z, \lambda)-x_{m-1}(t, z, \lambda)\right|, m=1,2, \ldots .
$$

According to the recurrence relation (3.10) of Lemma 2, using the Lipschitz condition $f \in \operatorname{Lip}\left(K_{x}, D^{x}\right)$ and estimation (3.11), for $m=1$ it follows from (5.12) and (5.10) that

$$
r_{2}(t, z, \lambda) \leq K_{x}\left[\left(1-\frac{2(t-a)}{b-a}\right) \int_{a}^{t} \alpha_{1}\left(s, a, \frac{a+b}{2}\right) d s+\right.
$$




$$
\begin{array}{r}
\left.+\frac{2(t-a)}{b-a} \int_{t}^{b} \alpha_{1}\left(s, a, \frac{a+b}{2}\right) d s\right] \delta_{\left[a, \frac{a+b}{2}\right], D^{x}}(f) \leq \quad \text { (5.14) } \\
\leq K_{x} \alpha_{2}\left(t, a, \frac{a+b}{2}\right) \delta_{\left[a, \frac{a+b}{2}\right], D^{x}}(f) \leq \frac{10}{9} Q_{x} \alpha_{1}\left(t, a, \frac{a+b}{2}\right) \delta_{\left[a, \frac{a+b}{2}\right], D^{x}}(f),
\end{array}
$$

where the matrix $Q_{x}$ has the form given in (5.1). By induction we can easily establish that

$$
\begin{aligned}
r_{m+1}(t, z, \lambda) \leq K_{x}^{m} \alpha_{m+1}(t, & \left.a, \frac{a+b}{2}\right) \delta_{\left[a, \frac{a+b}{2}\right], D^{x}}(f) \leq \\
& \leq \frac{10}{9} Q_{x}^{m} \alpha_{1}\left(t, a, \frac{a+b}{2}\right) \delta_{\left[a, \frac{a+b}{2}\right], D^{x}}(f),
\end{aligned}
$$

Therefore, in view of (5.15)

$$
\begin{gathered}
\left|x_{m+j}(t, z, \lambda)-x_{m}(t, z, \lambda)\right| \leq \\
\leq\left|x_{m+j}(t, z, \lambda)-x_{m+j-1}(t, z, \lambda)\right|+\left|x_{m+j-1}(t, z, \lambda)-x_{m+j-2}(t, z, \lambda)\right|+\ldots+ \\
+\left|x_{m+1}(t, z, \lambda)-x_{m}(t, z, \lambda)\right|=\sum_{i=1}^{j} r_{m+i}(t, z, \lambda) \leq \\
\leq \frac{10}{9} \alpha_{1}\left(t, a, \frac{a+b}{2}\right) \sum_{i=1}^{j} Q_{x}^{m+i-1} \delta_{\left[a, \frac{a+b}{2}\right], D^{x}}(f)= \\
=\frac{10}{9} \alpha_{1}\left(t, a, \frac{a+b}{2}\right) Q_{x}^{m} \sum_{i=0}^{j-1} Q^{i} \delta_{\left[a, \frac{a+b}{2}\right], D^{x}}(f)
\end{gathered}
$$

where $\delta\left[a, \frac{a+b}{2}\right], D^{x}(f)$ is given according to (2.7). Since, due to (5.2), the maximum eigenvalue of the matrix $Q_{x}$ does not exceed the unity, we have

$$
\sum_{i=0}^{j-1} Q_{x}^{i} \leq\left(1_{n}-Q_{x}\right)^{-1}, \lim _{m \rightarrow \infty} Q_{x}^{m}=0_{n} .
$$

Therefore, we conclude from (5.16) that, according to Cauchy criterium, the sequence $\left\{x_{m}(t, z, \lambda)\right\}_{m=0}^{\infty}$ of the form (4.4) uniformly converges in the domain $(t, z, \lambda) \in$ $\left[a, \frac{a+b}{2}\right] \times D_{a} \times D_{\frac{a+b}{2}}$ to the limit function $x_{\infty}(t, z, \lambda)$. Since all functions of the sequence (4.4) satisfy the boundary conditions (1.7) for all values of the introduced parameters $z \in D_{a}, \eta \in D_{\frac{a+b}{2}}$ the limit function $x_{\infty}(t, z, \lambda)$ also satisfies these conditions. Passing to the limit as $m \rightarrow \infty$ in equality (4.4) we show that the limit function satisfies both the integral equation (5.5) and the Cauchy problem (5.6), 
(5.7), where $\Delta(z, \lambda)$ is given by (5.8). Passing to the limit as $j \rightarrow \infty$ in (5.16) we get the estimation (5.9).

By analogy with Theorem 1, under similar conditions, we can establish the uniform convergence of sequence (5.8).

Theorem 2. Let there exist a non negative vector $\rho^{y}$ such that $f \in \operatorname{Lip}\left(K_{y}, D^{y}\right)$ on the interval $t \in\left[\frac{a+b}{2}, b\right]$ with $\rho^{y}$ satisfying the inequality (4.5) and for the matrix

$$
Q_{y}:=\frac{3 T}{20} K_{y}
$$

the condition

$$
r\left(Q_{y}\right)<1
$$

is satisfied.

Then, for arbitrary fixed pair of vectors $(\lambda, \eta) \in D_{\frac{a+b}{2}} \times D_{b}$ :

1. All members of sequence (4.8) are continuously differentiable functions on the interval $t \in\left[\frac{a+b}{2}, b\right]$ satisfying the two-point separated parameterized boundary conditions (1.9).

2. The sequence of functions (4.8) in $t \in\left[\frac{a+b}{2}, b\right]$ converges uniformly as $m \rightarrow \infty$ to the limit function

$$
y_{\infty}(t, \lambda, \eta)=\lim _{m \rightarrow \infty} y_{m}(t, \lambda, \eta)
$$

3. The limit function satisfies the conditions

$$
y_{\infty}\left(\frac{a+b}{2}, \lambda, \eta\right)=\lambda, y_{\infty}(b, \lambda, \eta)=\eta .
$$

4. The function $y_{\infty}(t, \lambda, \eta)$ is a unique continuously differentiable solution of the integral equation

$$
\begin{aligned}
y(t)=\lambda+\int_{\frac{a+b}{2}}^{t} f(s, y(s)) d s-\frac{2 t-a-b}{2 b-a-b} \int_{\frac{a+b}{2}}^{b} f(s, y(s)) d s+ & \\
& +\frac{2 t-a-b}{2 b-a-b}[\eta-\lambda], t \in\left[\frac{a+b}{2}, b\right]
\end{aligned}
$$

in the domain $D^{y}$. In other words, $y_{\infty}(t, \lambda, \eta)$ is a solution of the following Cauchy problem for the modified system of integro-differential equations:

$$
\frac{d y}{d t}=f(t, y)+\frac{2}{2 b-a-b} H(\lambda, \eta), t \in\left[\frac{a+b}{2}, b\right]
$$




$$
y\left(\frac{a+b}{2}\right)=\lambda
$$

where $H(\lambda, \eta): D_{\frac{a+b}{2}} \times D_{b} \rightarrow \mathbb{R}^{n}$ is a mapping given by the formula

$$
H(\lambda, \eta)=\eta-\lambda-\int_{\frac{a+b}{2}}^{b} f\left(s, y_{\infty}(s, \lambda, \eta)\right) d s .
$$

5. The following estimate holds

$$
\begin{gathered}
\left|y_{\infty}(\cdot, \lambda, \eta)-y_{m}(\cdot, \lambda, \eta)\right| \\
\leqslant \frac{10}{9} \alpha_{1}\left(t, \frac{a+b}{2}, \frac{b-a}{2}\right) Q_{y}^{m}\left(1_{n}-Q_{y}\right)^{-1} \delta_{\left[\frac{a+b}{2}, b\right] \times D^{y}}(f), \\
t \in\left[\frac{a+b}{2}, b\right], m \geq 0,
\end{gathered}
$$

where $\delta_{\left[\frac{a+b}{2}, b\right] \times D^{y}}(f)$ is given in (2.7) and $\alpha_{1}\left(t, \frac{a+b}{2}, \frac{b-a}{2}\right)$ is defined by (3.9).

Proof. The proof can be carried out similarly to the proof of Theorem 1.

\section{LIMIT FUNCTIONS AND DETERMINING EQUATIONS}

It is natural to expect that the limit functions $x_{\infty}(t, z, \lambda)$ and $y_{\infty}(t, \lambda, \eta)$ of the iterations (4.4) and (4.8) on the half-intervals will help one to formulate criteria of solvability of the integral BVP (1.4), (1.5). It turns out that the functions

$$
\Delta(z, \lambda): D_{a} \times D_{\frac{a+b}{2}} \rightarrow \mathbb{R}^{n} \text { and } H(\lambda, \eta): D_{\frac{a+b}{2}} \times D_{b} \rightarrow \mathbb{R}^{n}
$$

defined according to equalities (5.8) and (5.25) provide such conclusion.

Indeed, Theorems 1 and 2 guarantee that under the conditions assumed, the functions

$$
x_{\infty}(t, z, \lambda):\left[a, \frac{a+b}{2}\right] \rightarrow \mathbb{R}^{n} \text { and } y_{\infty}(t, \lambda, \eta):\left[\frac{a+b}{2}, b\right] \rightarrow \mathbb{R}^{n}
$$

are well defined for all $(z, \lambda) \in D_{a} \times D_{\frac{a+b}{2}}$ and $(\lambda, \eta) \in D_{\frac{a+b}{2}} \times D_{b}$. Therefore, by putting

$$
u_{\infty}(t, z, \lambda, \eta):=\left\{\begin{array}{l}
x_{\infty}(t, z, \lambda), \text { if } t \in\left[a, \frac{a+b}{2}\right] \\
y_{\infty}(t, \lambda, \eta), \text { if } t \in\left[\frac{a+b}{2}, b\right]
\end{array}\right.
$$

we obtain a function $u_{\infty}(\cdot, z, \lambda, \eta):[a, b] \rightarrow \mathbb{R}^{n}$, which is well defined for the same values $(z, \lambda) \in D_{a} \times D_{\frac{a+b}{2}}$ and $(\lambda, \eta) \in D_{\frac{a+b}{2}} \times D_{b}$. This function is obviously 
continuous, because at the point $t=\frac{a+b}{2}$ we have

$$
x_{\infty}\left(\frac{a+b}{2}, z, \lambda\right)=y_{\infty}\left(\frac{a+b}{2}, \lambda, \eta\right)=\lambda .
$$

Along with equations (1.6) and (1.8) defined on the intervals $\left[a, \frac{a+b}{2}\right]$ and $\left[\frac{a+b}{2}, b\right]$ respectively, consider the following equations with the additive perturbation of the right side

$$
\frac{d x}{d t}=f(t, x)+\frac{2}{b-a} \mu^{x}, t \in\left[a, \frac{a+b}{2}\right]
$$

with initial condition

$$
x(a)=z,
$$

and

$$
\frac{d y}{d t}=f(t, y)+\frac{2}{2 b-a-b} \mu^{y}, t \in\left[\frac{a+b}{2}, b\right]
$$

with initial condition

$$
y\left(\frac{a+b}{2}\right)=\lambda
$$

where

$$
\mu^{x}=\operatorname{col}\left(\mu_{1}^{x}, \ldots, \mu_{n}^{x}\right), \mu^{y}=\operatorname{col}\left(\mu_{1}^{y}, \ldots, \mu_{n}^{y}\right) \in \mathbb{R}^{n}
$$

are some control parameters.

Theorem 3. Let $z \in D_{a}$ and $\lambda \in D_{\frac{a+b}{2}}$ be fixed. Suppose that all conditions of Theorems 1,2 hold.

Then, for the solutions $x(\cdot, a, z)$ and $y\left(\cdot, \frac{a+b}{2}, \lambda\right)$ of the Cauchy problems (6.5), (6.6) and (6.7),(6.8), to have the properties

$$
x\left(\frac{a+b}{2}, a, z\right)=\lambda
$$

and

$$
y\left(b, \frac{a+b}{2}, \lambda\right)=\eta
$$

respectively, by other words, to satisfy the parameterized separated two-point boundary conditions (1.7) and (1.9), respectively, it is necessary and sufficient that the control parameters $\mu^{x}$ and $\mu^{y}$ be given by the formulas

$$
\mu^{x}:=\lambda-z-\int_{a}^{\frac{a+b}{2}} f\left(s, x_{\infty}(s, z, \lambda)\right) d s
$$


and

$$
\mu^{y}:=\eta-\lambda-\int_{\frac{a+b}{2}}^{b} f\left(s, y_{\infty}(s, \lambda, \eta)\right) d s,
$$

where $x_{\infty}(\cdot, z, \lambda)$ and $y_{\infty}(\cdot, \lambda, \eta)$ are the limit functions of the sequences (4.4) and (4.8), respectively. Moreover, in this case

$$
x(\cdot, a, z)=x_{\infty}(\cdot, z, \lambda)
$$

and

$$
y\left(\cdot, \frac{a+b}{2}, \lambda\right)=y_{\infty}(\cdot, \lambda, \eta)
$$

Proof. The proof can be carried out similarly to the proof of Theorem 2 from [9].

The following theorem establishes a relation of function (6.3) to the solution of integral BVP (1.4), (1.5) in terms of the zeros of functions $\Delta(z, \lambda): D_{a} \times D_{\frac{a+b}{2}} \rightarrow$ $\mathbb{R}^{n}$ and $H(\lambda, \eta): D_{\frac{a+b}{2}} \times D_{b} \rightarrow \mathbb{R}^{n}$, defined according to (5.8) and (5.25).

Theorem 4. Let the conditions of Theorem 1,2 hold. Then :

1. The function $u_{\infty}(\cdot, z, \lambda, \eta):[a, b] \rightarrow \mathbb{R}^{n}$ defined by (6.3) is a continuously differentiable solution of the boundary value problem (1.4), (1.5) if and only if the triplet $(z, \lambda, \eta)$ satisfies the system of $3 n$ algebraic equations

$$
\begin{gathered}
\Delta(z, \lambda)=\lambda-z-\int_{a}^{\frac{a+b}{2}} f\left(s, x_{\infty}(t, z, \lambda)\right) d s=0, \\
H(\lambda, \eta)=\eta-\lambda-\int_{\frac{a+b}{2}}^{b} f\left(s, y_{\infty}(s, \lambda, \eta)\right) d s=0, \\
P(z, \lambda, \eta):=\int_{a}^{\frac{a+b}{2}} g\left(s, x_{\infty}(s, z, \lambda)\right) d s+\int_{\frac{a+b}{2}}^{b} g\left(s, y_{\infty}(s, \lambda, \eta)\right) d s-d=0 .
\end{gathered}
$$

2. For every solution $U(\cdot)$ of problem (1.4), (1.5) with $\left(U(a), U\left(\frac{a+b}{2}\right), U(b)\right)$ $\in D_{a} \times D_{\frac{a+b}{2}} \times D_{b}$, there exist a triplet $\left(z_{0}, \lambda_{0}, \eta_{0}\right)$ such that $U(\cdot)=u_{\infty}\left(t, z_{0}, \lambda_{0}\right.$, $\left.\eta_{0}\right)$, where the function $u_{\infty}\left(t, z_{0}, \lambda_{0}, \eta_{0}\right)$ is given in (6.3). 
Proof. The proof can be carried out similarly to the proof of Theorem 4 from [4]. The continuous differentiability of the solution $u_{\infty}(\cdot, z, \lambda, \eta):[a, b] \times \mathbb{R}^{n} \times \mathbb{R}^{n} \times$ $\mathbb{R}^{n} \rightarrow \mathbb{R}^{n}$ at the point $t=\frac{a+b}{2}$ follows from the equations (6.4), (5.6), (5.23), (6.16), (6.17) and the continuous differentiability of this function at other points is obvious from its definition.

Equations (6.16) are usually referred to as determining or bifurcation equations because their roots determine solutions of the original problem.

\section{APPROXIMATE DETERMINING EQUATIONS}

Although Theorem 4 provides a theoretical answer to the question on the construction of a solution of the boundary value problem (1.4), (1.5), its application faces difficulties due to the fact that the explicit form of $x_{\infty}(t, z, \lambda), y_{\infty}(t, \lambda, \eta)$ and the functions

$$
\begin{gathered}
\Delta(z, \lambda): D_{a} \times D_{\frac{a+b}{2}} \rightarrow \mathbb{R}^{n}, H(\lambda, \eta): D_{\frac{a+b}{2}} \times D_{b} \rightarrow \mathbb{R}^{n}, \\
P(z, \lambda, \eta): D_{a} \times D_{\frac{a+b}{2}} \times D_{b} \rightarrow \mathbb{R},
\end{gathered}
$$

appearing in (6.16), (6.17), (6.18) are usually unknown. This complication can be overcome by using $x_{m}(s, z, \lambda), y_{m}(s, \lambda, \eta)$ for a fixed $m$, which will lead one to the so-called approximate determining equations:

$$
\begin{gathered}
\Delta_{m}(z, \lambda)=\lambda-z-\int_{a}^{\frac{a+b}{2}} f\left(s, x_{m}(t, z, \lambda)\right) d s=0, \\
H_{m}(\lambda, \eta)=\eta-\lambda-\int_{\frac{a+b}{2}}^{b} f\left(s, y_{m}(s, \lambda, \eta)\right) d s=0, \\
P_{m}(z, \lambda, \eta):=\int_{a}^{\frac{a+b}{2}} g\left(s, x_{m}(s, z, \lambda)\right) d s+\int_{\frac{a+b}{2}}^{b} g\left(s, y_{m}(s, \lambda, \eta)\right) d s-d=0 .
\end{gathered}
$$

Note that, unlike system (6.16), (6.17), (6.18), the $m$-th approximate determining system (7.1), (7.2), (7.3) contains only terms involving the functions $x_{m}(\cdot, z, \lambda)$, $y_{m}(\cdot, \lambda, \eta)$, and thus it is known explicitly.

It is natural to expect that approximations to the unknown solution of problems (1.4), (1.5) can be obtained by using the function

$$
u_{m}(t, z, \lambda, \eta):=\left\{\begin{array}{l}
x_{m}(t, z, \lambda), \text { if } t \in\left[a, \frac{a+b}{2}\right] \\
y_{m}(t, \lambda, \eta), \text { if } t \in\left[\frac{a+b}{2}, b\right]
\end{array}\right.
$$


which is an "approximate" version of (6.3) and well defined for all $t \in[a, b]$ and $(z, \lambda) \in D_{a} \times D_{\frac{a+b}{2}},(\lambda, \eta) \in D_{\frac{a+b}{2}} \times D_{b}$.

Lemma 3. If $z, \lambda$ and $\eta$ satisfy equations (7.1), (7.2) and (7.3) for a certain $m$, then the function $u_{m}(t, z, \lambda, \eta)$ determined by equality (7.4) is continuously differentiable on $[a, b]$.

Proof. We recall that the functions of the sequences (4.4) and (4.8) have the property

$$
x_{m}\left(\frac{a+b}{2}, z, \lambda\right)=y_{m}\left(\frac{a+b}{2}, \lambda, \eta\right)=\lambda .
$$

It follows immediately from (4.4), (4.8) that

$$
\begin{gathered}
\frac{d x_{m+1}\left(\frac{a+b}{2}, z, \lambda\right)}{d t}=f\left(\frac{a+b}{2}, x_{m}\left(\frac{a+b}{2}, z, \lambda\right)\right)- \\
-\frac{2}{b-a} \int_{a}^{\frac{a+b}{2}} f\left(s, x_{m}(s, z, \lambda)\right) d s+\frac{2}{b-a}[\lambda-z]
\end{gathered}
$$

and

$$
\begin{gathered}
\frac{d y_{m+1}\left(\frac{a+b}{2}, \lambda, \eta\right)}{d t}=f\left(\frac{a+b}{2}, y_{m}\left(\frac{a+b}{2}, \lambda, \eta\right)\right)- \\
-\frac{2}{2 b-a-b} \int_{\frac{a+b}{2}}^{b} f\left(s, y_{m}(s, z, \lambda)\right) d s+\frac{2}{2 b-a-b}[\eta-\lambda] .
\end{gathered}
$$

In view of (7.1) and (7.2) it follows from (7.6) and (7.7) that

$$
\frac{d x_{m+1}\left(\frac{a+b}{2}, z, \lambda\right)}{d t}=f\left(\frac{a+b}{2}, x_{m}\left(\frac{a+b}{2}, z, \lambda\right)\right)
$$

and

$$
\frac{d y_{m+1}\left(\frac{a+b}{2}, \lambda, \eta\right)}{d t}=f\left(\frac{a+b}{2}, y_{m}\left(\frac{a+b}{2}, \lambda, \eta\right)\right) .
$$

In view of (7.5) it follows from (7.8), (7.9) that

$$
\frac{d x_{m+1}\left(\frac{a+b}{2}, z, \lambda\right)}{d t}=\frac{d y_{m+1}\left(\frac{a+b}{2}, \lambda, \eta\right)}{d t}
$$

and therefore according to (7.4) $\frac{d \bar{u}_{m+1}(\cdot, z, \lambda, \eta)}{d t}$ is continuous at $\frac{a+b}{2}$. The continuous differentiability of the function $u_{m}(\cdot, z, \lambda, \eta)$ in other points is obvious from its definition. 


\section{APPROXIMATION OF THE SOLUTIONS}

Theorem 4 can be complemented by the following natural observation. Let $(\widetilde{z}, \widetilde{\lambda}, \widetilde{\eta}) \in D_{a} \times D_{\frac{a+b}{2}} \times D_{b}$ be a root of the approximate determining system (7.1), (7.2), (7.3) for a certain $m$. Then the function

$$
U_{m}(t)=u_{m}(t, \widetilde{z}, \widetilde{\lambda}, \widetilde{\eta}):=\left\{\begin{array}{c}
x_{m}(t, \widetilde{z}, \widetilde{\lambda}), \text { if } t \in\left[a, \frac{a+b}{2}\right] \\
y_{m}(t, \widetilde{\lambda}, \widetilde{\eta}), \text { if } t \in\left[\frac{a+b}{2}, b\right],
\end{array},\right.
$$

defined according to (7.4) can be regarded as the $m-$ th approximation to a solution of the two-point boundary value problem (1.4), (1.5). This is justified by Lemma 3 . The following estimates follow directly from Theorems 1,2

$$
\begin{gathered}
\left|\widetilde{x}_{\infty}(t, \widetilde{z}, \widetilde{\lambda})-x_{m}(t, \widetilde{z}, \widetilde{\lambda})\right| \leq \\
\leq \frac{10}{9} \alpha_{1}\left(t, a, \frac{b-a}{2}\right) Q_{x}^{m}\left(1_{n}-Q_{x}\right)^{-1} \delta_{\left[a, \frac{a+b}{2}\right] \times D^{x}}(f), t \in\left[a, \frac{a+b}{2}\right], \quad \\
\left|\widetilde{y}_{\infty}(t, \widetilde{\lambda}, \widetilde{\eta})-y_{m}(t, \widetilde{\lambda}, \widetilde{\eta})\right| \leq \\
\leq \frac{10}{9} \alpha_{1}\left(t, \frac{a+b}{2}, \frac{b-a}{2}\right) Q_{y}^{m}\left(1_{n}-Q_{y}\right)^{-1} \delta_{\left[\frac{a+b}{2}, b\right] \times D^{y}}(f), t \in\left[a, \frac{a+b}{2}\right] .
\end{gathered}
$$

\section{EXAMPLE}

Let us apply the numerical-analytic approach described above to the system of differential equations

$$
\left\{\begin{array}{l}
x_{1}^{\prime}(t)=\frac{t}{4} x_{2}^{2}(t)-\frac{7 t}{2} x_{1}(t)+\frac{27}{64} t^{3}+\frac{3}{5} t \\
x_{2}^{\prime}(t)=t^{2} x_{1}(t)-\frac{t}{4} x_{2}(t)-\frac{1}{8} t^{4}-\frac{3}{80} t^{2}+\frac{1}{4}, t \in[0,1],
\end{array}\right.
$$

considered with the integral boundary conditions

$$
\left\{\begin{array}{c}
\int_{0}^{1} s x_{1}(s) x_{2}(s) d s=\frac{7}{480} \\
\int_{0}^{1} s^{2} x_{2}^{2}(s) d s=\frac{1}{80}
\end{array} .\right.
$$

Clearly, (9.1) is a particular case of (1.4), (1.5) with $a:=0, b:=1$,

$$
\begin{gathered}
f\left(t, x_{1}, x_{2}\right):=\left(\begin{array}{c}
\frac{t}{4} x_{2}^{2}-\frac{7 t}{2} x_{1}+\frac{27}{64} t^{3}+\frac{3}{5} t, \\
t^{2} x_{1}-\frac{t}{4} x_{2}-\left(\frac{1}{8} t^{2}-\frac{3}{80}\right) t^{2}+\frac{1}{4}
\end{array}\right), \\
g\left(t, x_{1}, x_{2}\right):=\left(\begin{array}{c}
t x_{1} x_{2} \\
t^{2} x_{2}^{2}
\end{array}\right) \text {, and } d:=\left(\begin{array}{c}
7 / 480 \\
1 / 80
\end{array}\right) .
\end{gathered}
$$


It is easy to check that

$$
x_{1}^{*}(t)=\frac{t^{2}}{8}+\frac{1}{10}, x_{2}^{*}(t)=\frac{t}{4}
$$

is a continuously differentiable solution of the problem (9.1), (9.2).

Following (1.7) and (1.9), introduce parameters

$$
\begin{gathered}
z:=x(0)=\operatorname{col}\left(x_{1}(0), x_{2}(0)\right)=\operatorname{col}\left(z_{1}, z_{2}\right), \\
\lambda:=x\left(\frac{1}{2}\right)=\operatorname{col}\left(x_{1}\left(\frac{1}{2}\right), x_{2}\left(\frac{1}{2}\right)\right)=\operatorname{col}\left(\lambda_{1}, \lambda_{2}\right), \\
\eta:=x(1)=\operatorname{col}\left(x_{1}(1), x_{2}(1)\right)=\operatorname{col}\left(\eta_{1}, \eta_{2}\right) .
\end{gathered}
$$

Let us choose the sets $D_{a}$ and $D_{\frac{a+b}{2}}$, where one looks for the values $x(a)$ and $x\left(\frac{a+b}{2}\right)$, as follows:

$$
D_{a}=D_{\frac{a+b}{2}}=\left\{\left(x_{1}, x_{2}\right): 0.001 \leq x_{1} \leq 0.14,-0.1 \leq x_{2} \leq 0.14\right\} .
$$

Let us choose the sets $D_{\frac{a+b}{2}}$ and $D_{b}$, where one looks for the values $x\left(\frac{a+b}{2}\right)$ and $x(b)$ and as follows:

$$
D_{\frac{a+b}{2}}=D_{b}=\left\{\left(y_{1}, y_{2}\right): 0.12 \leq y_{1} \leq 0.23,0.1 \leq y_{2} \leq 0.26\right\} .
$$

In this case, a convex linear combination $D^{x}$ of form (3.1) of vectors $z \in D_{a}$ and $\lambda \in D_{\frac{a+b}{2}}$ will be $D^{x}=D_{a}=D_{\frac{a+b}{2}}$.

In this case, a convex linear combination $D^{y}$ of form (3.3) of vectors $\lambda \in D_{\frac{a+b}{2}}$ and $\eta \in D_{b}$ will be $D^{y}=D_{\frac{a+b}{2}}=D_{b}$.

For $\rho^{x}, \rho^{y}$ involved in (3.3), (3.5) and (4.1), (4.5) we choose the value

$$
\rho^{x}=\rho^{y}:=\operatorname{col}(0.7 ; 0.7) .
$$

Consequently $\rho^{x}$-neighbourhood $D^{x}$ of the set $D_{a, \frac{a+b}{2}}$ is given as follows

$$
D^{x}=\left\{\left(x_{1}, x_{2}\right):-0.701 \leq x_{1} \leq 0.84,-0.8 \leq x_{2} \leq 0.84\right\}
$$

Consequently $\rho^{y}$-neighbourhood $D^{y}$ of the set $D_{\frac{a+b}{2}, b}$ is given as follows

$$
D^{y}=\left\{\left(y_{1}, y_{2}\right):-0.58 \leq y_{1} \leq 0.93,-0.6 \leq y_{2} \leq 0.96\right\} .
$$

Direct computations show that the Lipschitz condition (2.5) for the right hand side in (9.1) in the domain $D$ holds with matrix

$$
K=\left[\begin{array}{cc}
7 / 2 & 1 / 8 \\
1 & 1 / 4
\end{array}\right]
$$

and

$$
Q=\frac{3}{10}\left[\begin{array}{cc}
7 / 2 & 1 / 8 \\
1 & 1 / 4
\end{array}\right], r(Q)=1.061405>1,
$$




$$
\begin{aligned}
& Q_{x}:=\frac{3}{20} K_{x}=\frac{3}{20}\left[\begin{array}{cc}
7 / 4 & 1 / 32 \\
1 / 4 & 1 / 8
\end{array}\right], \quad r\left(Q_{x}\right)=0.2632<1, \\
& Q_{y}:=\frac{3}{20} K_{y}=\frac{3}{20}\left[\begin{array}{cc}
7 / 2 & 1 / 8 \\
1 & 1 / 4
\end{array}\right], \quad r\left(Q_{y}\right)=0.5307<1, \\
& \delta_{\left[a, \frac{a+b}{2}\right], D^{x}}(f):=\frac{1}{2}\left[\max _{(t, x) \in\left[0, \frac{1}{2}\right] \times D^{x}} f(t, x)-\min _{(t, x) \in\left[0, \frac{1}{2}\right] \times D^{x}} f(t, x)\right] \\
& =\left[\begin{array}{l}
1.392475 \\
0.295125
\end{array}\right] \text {, } \\
& \rho^{x}=\left[\begin{array}{l}
0.7 \\
0.7
\end{array}\right] \geq \frac{b-a}{4} \delta_{\left[a, \frac{a+b}{2}\right], D^{x}}(f)=\left[\begin{array}{c}
0.34811875, \\
0.07378125
\end{array}\right] \text {. } \\
& \delta_{\left[\frac{a+b}{2}, b\right], D^{y}}(f):=\frac{1}{2}\left[\max _{(t, x) \in\left[\frac{1}{2}, 1\right] \times D^{y}} f(t, x)-\min _{(t, x) \in\left[\frac{1}{2}, 1\right] \times D^{y}} f(t, x)\right] \\
& =\left[\begin{array}{c}
2.7577 \\
0.95
\end{array}\right] \text {, } \\
& \rho^{y}=\left[\begin{array}{l}
0.7 \\
0.7
\end{array}\right] \geq \frac{b-a}{4} \delta_{\left[\frac{a+b}{2}, b\right], D^{y}}(f)=\left[\begin{array}{c}
0.689425, \\
0.2375
\end{array}\right] .
\end{aligned}
$$

So, we check that all conditions of Theorems 1,2 are fulfill, and the sequence of functions (4.1) for this example is convergent.

Using (4.4),(4.7) and applying Maple 14 at the first iteration $(m=1)$ we obtain the following results for the first and the second component:

$$
\begin{gathered}
x_{11}(t, z, \lambda):=z_{1}+1 / 4\left(1 / 4\left(-2 z_{2}+2 \lambda_{2}\right)^{2}+27 / 64\right) t^{4}+1 / 3\left(7 z_{1}-7 \lambda_{1}+\right. \\
\left.+1 / 2 z_{2}\left(-2 z_{2}+2 \lambda_{2}\right)\right) t^{3}+1 / 2\left(1 / 4 z_{2}^{2}+3 / 5-7 / 2 z_{1}\right) t^{2}-2 t((1 / 256) \times \\
\left(-2 z_{2}+2 \lambda_{2}\right)^{2}+1671 / 20480-(7 / 48) z_{1}-(7 / 24) \lambda_{1}+ \\
\left.+(1 / 48) z_{2}\left(-2 z_{2}+2 \lambda_{2}\right)+(1 / 32) z_{2}^{2}\right)+2 t\left(\lambda_{1}-z_{1}\right), \\
x_{12}(t, z, \lambda):=z_{2}+1 / 4 t-1 / 40 t^{5}+1 / 4\left(-2 z_{1}+2 \lambda_{1}\right) t^{4}+ \\
+1 / 3\left(1 / 2 z_{2}-1 / 2 \lambda_{2}+z_{1}-3 / 80\right) t^{3}-1 / 8 z_{2} t^{2}-2 t(157 / 1280+ \\
\left.+(1 / 96) z_{1}+(1 / 32) \lambda_{1}-(1 / 96) z_{2}-(1 / 48) \lambda_{2}\right)+2 t\left(\lambda_{2}-z_{2}\right), \\
y_{11}(t, \lambda, \eta):=\lambda_{1}+1 / 4\left(1 / 4\left(-2 \lambda_{2}+2 \eta_{2}\right)^{2}+27 / 64\right)\left(t^{4}-1 / 16\right)+ \\
+1 / 3\left(7 \lambda_{1}-7 \eta_{1}+1 / 2\left(2 \lambda_{2}-\eta_{2}\right)\left(-2 \lambda_{2}+2 \eta_{2}\right)\right) t^{3}-1 / 8+(1 / 2((1 / 4) \times \\
\left.\left.\left(2 \lambda_{2}-\eta_{2}\right)^{2}+3 / 5-7 \lambda_{1}+(7 / 2) \eta_{1}\right)\right)\left(t^{2}-1 / 4\right)-(2 t-1)\left(( 1 5 / 2 5 6 ) \left(-2 \lambda_{2}+\right.\right.
\end{gathered}
$$




$$
\begin{gathered}
\left.+2 \eta_{2}\right)^{2}+6633 / 20480-(7 / 12) \lambda_{1}-(35 / 48) \eta_{1}+\left(7 / 48\left(2 \lambda_{2}-\eta_{2}\right)\right) \times \\
\left.\left(-2 \lambda_{2}+2 \eta_{2}\right)+(3 / 32)\left(2 \lambda_{2}-\eta_{2}\right)^{2}\right)+(2 t-1)\left(\eta_{1}-\lambda_{1}\right), \\
y_{12}(t, \lambda, \eta):=\lambda_{2}+1 / 4 t-159 / 1280-1 / 40 t^{5}+1 / 4\left(-2 \lambda_{1}+2 \eta_{1}\right)\left(t^{4}-1 / 16\right)+ \\
+1 / 3\left(1 / 2 \lambda_{2}-1 / 2 \eta_{2}+2 \lambda_{1}-\eta_{1}-3 / 80\right)\left(t^{3}-1 / 8\right)+ \\
+\left(1 / 2\left(-(1 / 2) \lambda_{2}+(1 / 4) \eta_{2}\right)\right)\left(t^{2}-1 / 4\right)-(2 t-1)\left(23 / 256+(11 / 96) \lambda_{1}+\right. \\
\left.+(17 / 96) \eta_{1}-(1 / 24) \lambda_{2}-(5 / 96) \eta_{2}\right)+(2 t-1)\left(\eta_{2}-\lambda_{2}\right) .
\end{gathered}
$$

The numerical computations show that the components of the solution of equations (7.1)-(7.3) for $m=1$ are:

$$
\begin{aligned}
& z_{1}:=z_{11} \approx 0.1000871447, \quad z_{2}:=z_{12} \approx-0.00003539691, \\
& \lambda_{1}:=\lambda_{11} \approx 0.1312562435, \quad \lambda_{2}:=\lambda_{12} \approx 0.1250395576, \\
& \eta_{1}:=\eta_{11} \approx 0.2248977438, \quad \eta_{2}:=\eta_{12} \approx 0.2500433008 .
\end{aligned}
$$

By putting (9.6) into (9.5), we obtain the first and the second components of the first approximation to the solution of the given integral BVP (9.1), (9.2).

The graphs of the first approximation and the exact solution (9.3) of the original boundary-value problem are shown on Figure 1.
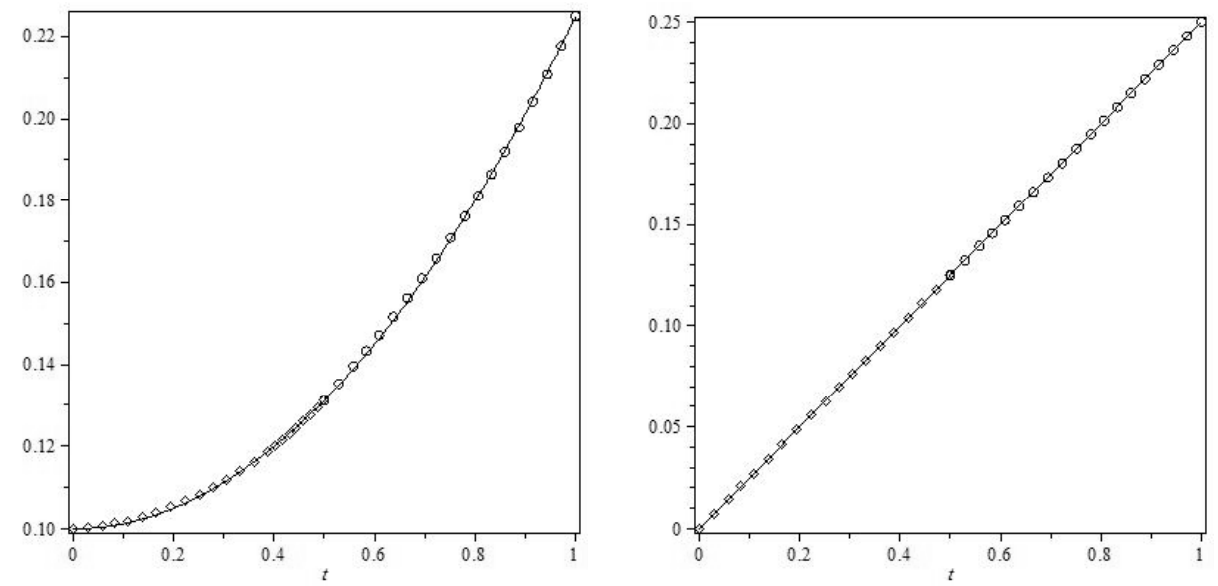

FIGURE 1. The components of the exact solution (solid line) and its first approximation (drawn with dots)

The number of the solutions of the algebraic determined system (5.23) is coincide with the number of the solutions of the given integral BVP. 
Let us choose the sets $D_{a}$ and $D_{\frac{a+b}{2}}$, where one looks for the values $x(a)$ and $x\left(\frac{a+b}{2}\right)$ as follows:

$$
D_{a}=D_{\frac{a+b}{2}}=\left\{\left(x_{1}, x_{2}\right):-0.47 \leq x_{1} \leq-0.2,-0.36 \leq x_{2} \leq-0.23\right\} .
$$

Let us choose the sets $D_{\frac{a+b}{2}}$ and, $D_{b}$ where one looks for the values $x\left(\frac{a+b}{2}\right)$ and $x(b)$ and as follows:

$$
\begin{gathered}
D_{\frac{a+b}{2}}=D_{b}=\left\{\left(y_{1}, y_{2}\right):-0.23 \leq y_{1} \leq 0.13,-0.25 \leq y_{2} \leq-0.12\right\} . \\
D^{x}=D_{a}=D_{\frac{a+b}{2}}, D^{y}=D_{\frac{a+b}{2}}=D_{b} .
\end{gathered}
$$

Consequently $\rho^{x}$-neighhourhood $D^{x}$ of the set $D_{a, \frac{a+b}{2}}$ is given as follows

$$
D^{x}=\left\{\left(x_{1}, x_{2}\right):-1.17 \leq x_{1} \leq 0.5,-1.06 \leq x_{2} \leq 0.47\right\} .
$$

Consequently $\rho^{y}$-neighhourhood $D^{y}$ of the set $D_{\frac{a+b}{2}, b}$ is given as follows

$$
\begin{gathered}
D^{y}=\left\{\left(y_{1}, y_{2}\right):-0.93 \leq y_{1} \leq 0.83,-0.95 \leq y_{2} \leq 0.58\right\}, \\
\delta_{\left[a, \frac{a+b}{2}\right], D^{x}}(f):=\frac{1}{2}\left[\begin{array}{c}
\left.\max _{(t, x) \in\left[0, \frac{1}{2}\right] \times D^{x}} f(t, x)-\min _{(t, x) \in\left[0, \frac{1}{2}\right] \times D^{x}} f(t, x)\right] \\
=\left[\begin{array}{c}
1.531475, \\
0.30437
\end{array}\right], \\
\rho^{x}=\left[\begin{array}{l}
0.7 \\
0.7
\end{array}\right] \geq \frac{b-a}{4} \delta_{\left[a, \frac{a+b}{2}\right], D^{x}}(f)=\left[\begin{array}{c}
0.3828687, \\
0.07609375
\end{array}\right], \\
\delta_{\left[\frac{a+b}{2}, b\right], D^{y}}(f):=\frac{1}{2}\left[\begin{array}{c}
\max _{(t, x) \in\left[\frac{1}{2}, 1\right] \times D^{y}} f(t, x)-\min _{(t, x) \in\left[\frac{1}{2}, 1\right] \times D^{y}} f(t, x)
\end{array}\right] \\
\rho^{y}=\left[\begin{array}{c}
2.6678125 \\
0.92125
\end{array}\right], \\
0.7
\end{array}\right] \geq \frac{b-a}{4} \delta_{\left[\frac{a+b}{2}, b\right], D^{y}}(f)=\left[\begin{array}{c}
0.6669531250, \\
0.2303125
\end{array}\right] .
\end{gathered}
$$

Computations show that the approximate determined system of algebraic equations (7.1)-(7.3) side by side with the solution (9.6) for $m=1$ has an another solution

$$
\begin{aligned}
& \hat{z}_{1}:=z_{11} \approx-0.4600459223, \quad \hat{z}_{2}:=z_{12} \approx-0.3588100487, \\
& \hat{\lambda}_{1}:=\lambda_{11} \approx-0.2288301475, \quad \hat{\lambda}_{2}:=\lambda_{12} \approx-0.2405391230, \\
& \hat{\eta}_{1}:=\eta_{11} \approx 0.1278495443, \quad \hat{\eta}_{2}:=\eta_{12} \approx-0.1346356173 .
\end{aligned}
$$

By substituting (9.7) into first approximation (9.5) we obtain the first approximation to the second solution of given integral BVP (9.1), (9.2). 
By analogy we obtain the second, third and fourth approximations $(m=2, m=$ $3, m=4)$

The graphs of the first and the fourth approximations to the second solution of the given BVP are shown on Figure 2.
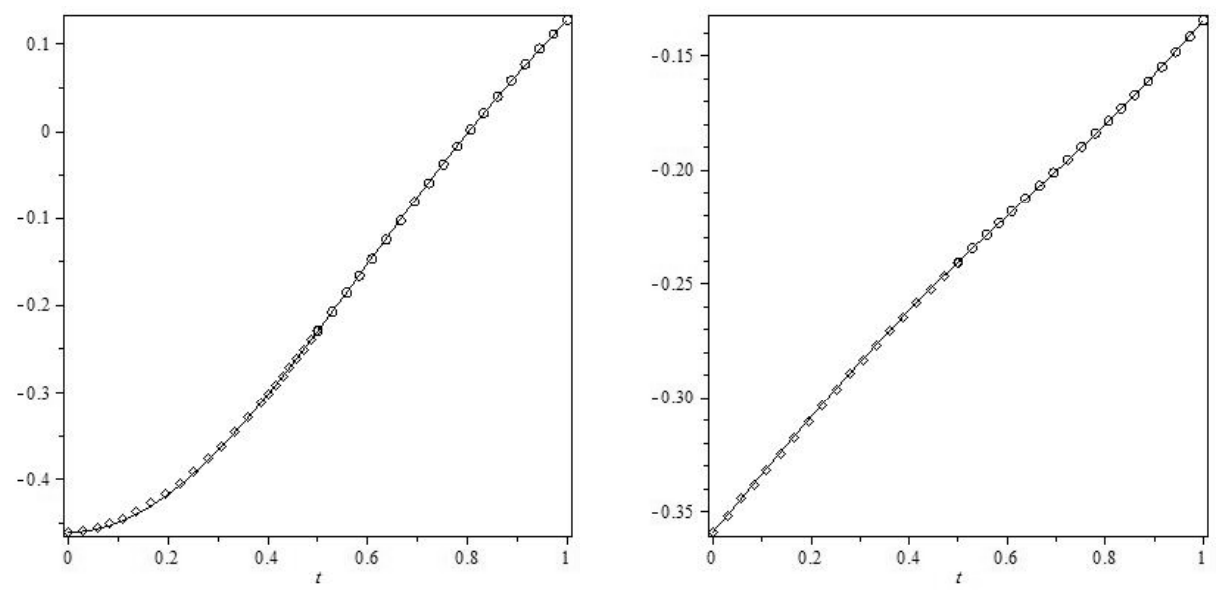

FIGURE 2. The components of the first (o) and the fourth (solid line) approximations to the second solution

\section{REFERENCES}

[1] M. Benchohra, J. Nieto, and A. Ouahab, "Second-order boundary value proble with integral boundary conditions," Bound. Value Probl., pp. 2011:260309, 9, 2011, doi: $10.1155 / 2011 / 260309$.

[2] J. Mao, Z. Zhao, and N. Xu, "On existence and uniqueness of positive solutions for integral boundary value problems," Electron. J. Qual. Theory Differ. Equ., no. 16, pp. 1-8, 2010.

[3] A. Rontó and M. Rontó, "Successive approximation techniques in non-linear boundary value problems for ordinary differential equations," in Handbook of differential equations: ordinary differential equations. Vol. IV, ser. Handb. Differ. Equ. Elsevier/North-Holland, Amsterdam, 2008, pp. 441-592, doi: 10.1016/S1874-5725(08)80010-7.

[4] A. Rontó and M. Rontó, "Periodic successive approximations and interval halving," Miskolc Math. Notes, vol. 13, no. 2, pp. 459-482, 2012.

[5] A. Rontó and Y. Varha, "Constructive existence analysis of solutions of non-linear integral boundary value problems," Miskolc Math. Notes, vol. 15, no. 2, pp. 725-742, 2012.

[6] A. Rontó, M. Rontó, and J. Varha, "A new approach to non-local boundary value problems for ordinary differential systems," Applied Mathematics and Computation, vol. 250, pp. 689-700, 2015, doi: 10.1016/j.amc.2014.11.021.

[7] A. Rontó, M. Rontó, and N. Shchobak, "Constructive analysis of periodic solutions with interval halving," Bound. Value Probl., pp. 2013:57, 34, 2013, doi: 10.1186/1687-2770-2013-57.

[8] A. Rontó, M. Rontó, and N. Shchobak, "Notes on interval halving procedure for periodic and twopoint problems," Bound. Value Probl., pp. 2014::164, 20, 2014, doi: 10.1186/s13661-014-0164-9. 
[9] M. Rontó, Y. Varha, and K. Marynets, "Further results on the investigation of solutions of integral boundary value problems," Tatra Mt. Math. Publ., vol. 63, pp. 247-267, 2015, doi: 10.1515/tmmp2015-0036.

[10] M. Rontó and K. Marynets, "Parametrization for non-linear problems with integral boundary conditions," Electron. J. Qual. Theory Differ. Equ., no. 99, pp. 1-23, 2012, doi: 10.14232/ejqtde.2012.1.99.

[11] M. Rontó and N. Shchobak, "On parametrization for a non-linear boundary value problem with separated conditions," Electron. J. Qual. Theory Differ. Equ., vol. 8, no. 18, pp. 1-16, 2007, doi: 10.14232/ejqtde.2007.7.18.

\section{Authors' addresses}

M. Rontó

Department of Analysis, University of Miskolc, 3515, Miskolc-Egyetemváros

E-mail address: matrontoduni-miskolc.hu

Y. Varha

Mathematical Faculty of Uzhgorod National University, 14 Universitetska St., 88000,Uzhgorod , Ukraine

E-mail address: jana.varha@mail.ru 